\title{
Prediction of Hepatic Clearance of Stereoselective Metabolism of Carvedilol in Liver Microsomes and Hepatocytes of Sprague-Dawley and Cytochrome P450 2D-Deficient Dark Agouti Rats
}

\author{
Masahiro Iwaki ${ }^{1,2,3}$, Toshiro Niwa ${ }^{1,4}$, Hiroyuki Tanaka ${ }^{1}$, Atsushi Kawase ${ }^{1}$, Hiroshi Komura ${ }^{1}$ \\ ${ }^{1}$ Department of Pharmacy, Faculty of Pharmacy, Kindai University, Osaka, Japan. ${ }^{2}$ Pharmaceutical Research and \\ Technology Institute, Kindai University, Osaka, Japan. ${ }^{3}$ Antiaging Center, Kindai University, Osaka, Japan. ${ }^{4}$ School of \\ Pharmacy, Shujitsu University, Okayama, Japan
}

Received, October 29, 2018; Revised, January 14, 2019; Accepted, January 16, 2019; Published, January 21, 2019.

\begin{abstract}
Hepatic clearance $\left(C L_{\mathrm{h}}\right)$ of carvedilol (CAR), which is eliminated via stereoselective metabolism by the CYP2D subfamily of cytochromes P450 (CYPs), was predicted using liver microsomes and hepatocytes from Sprague-Dawley (SD) rats and CYP2D-deficient Dark Agouti (DA) rats to determine the usefulness of prediction method. Plasma concentrations of CAR following intravenous injection to DA rats were higher than those in SD rats. The volume of distribution at steady state and total clearance $\left(C L_{\mathrm{tot}}\right)$ of $S$-CAR were approximately two times greater than those of $R$-CAR in both strains. $C L_{\mathrm{h}}$ predicted from in vitro studies using DA rat liver microsomes was different from that obtained from in vivo studies. In contrast, in vitro $C L_{\mathrm{h}}$ prediction using DA rat hepatocytes was nearly identical to the $C L_{h}$ observed in DA rats in vivo, and was lower than that in $\mathrm{SD}$ rats. The predicted $C L_{\mathrm{h}}$ in vitro using hepatocytes correlated well with the observed $C L_{\text {tot }}$ in vivo, which is expected to be nearly the same as $C L_{\mathrm{h}}$. These results suggest that in vitro metabolic studies using hepatocytes are more relevant with regard to stereoselectively predicting $C L_{\mathrm{h}}$ of CAR than those using liver microsomes.
\end{abstract}

\section{PURPOSE}

Carvedilol (CAR), a third-generation $\beta$ adrenoceptor antagonist that also blocks the $\beta_{1}$ adrenoreceptor (vasodilatation),(1) is used clinically as a racemic mixture of $R$ - and $S$-CAR, and exhibits enantioselective pharmacokinetic and pharmacodynamic properties.(2-5) Both enantiomers have equivalent $\alpha$-blocking activity, but the $\beta$-blocking activity of $S$-CAR is stronger than that of $R$-CAR. $(5,6)$ In humans, clearance of $R$-CAR is lower than the clearance of $S$-CAR, $(3,4)$ and is reduced further in poor metabolizers $(\mathrm{PM})$ with cytochrome P450 (CYP) 2D6 deficiency. $(2,3) R$ CAR is metabolized to form predominantly $4{ }^{\prime}-$ and 5'-hydroxy-CAR by polymorphic CYP2D6.(7)

For new drugs in early stages of development for which metabolic processes are not completely elucidated, older drugs for which metabolic profiles have not been reported, drugs for which metabolic pathways are multiple and complex, or drugs for which metabolites are not commercially available, the disappearance rate of a parent compound from an incubation medium is useful for identification of CYP isoform(s) responsible for metabolism and for assessment of linearity of pharmacokinetics.(8-11) We recently quantitatively characterized stereoselective biotransformation of CAR by human CYP isoforms by measuring disappearance rates of $R$-CAR and $S$-CAR in human liver microsomes and recombinant human CYPs. We showed that CYP2D2 predominantly catalyzed $R$-CAR metabolism, whereas CYP2D2 and CYP3A1/2 catalyzed $S$-CAR metabolism in Sprague Dawley (SD) rats.(12)

Dark Agouti (DA) rats exhibit lower mRNA levels of CYP2D1 and CYP2D2 than SD rats. Furthermore, DA rats have low metabolic activities toward CYP2D6 substrates. These findings suggest that DA and SD rats may be suitable animal models for PM and extensive metabolizers (EM) of CYP2D6 substrates, respectively.(13,14) However, previous studies in our laboratories have demonstrated a discrepancy between in vivo pharmacokinetic profiles in female DA rats and in vitro predicted profiles using liver microsomes for propranolol but not metoprolol.(15) In contrast, rat hepatocytes are useful for the prediction of in vivo pharmacokinetic profiles of some drugs metabolized

Corresponding Author: Masahiro Iwaki, Ph.D., Department of Pharmacy, Faculty of Pharmacy, Kindai University, 3-4-1 Kowakae, Higashi-osaka, Osaka 577-8501, Japan. E-mail: iwaki@phar.kindai.ac.jp 
by phase II enzymes. $(16,17)$ Prediction of hepatic clearance $\left(C L_{\mathrm{h}}\right)$ in vitro using liver microsomes should use a multi-dimensional approach, including metabolic experiments evaluating CYP and/or phase II enzymes such as UDP-glucuronosyltransferase (UGT) and protein binding to plasma and microsomal proteins. However, in vitro metabolic studies using hepatocytes cultured with plasma do not require estimation of protein binding for the prediction of $C L_{\mathrm{h}}$ values. Furthermore, as CAR is metabolized by CYPs and UGT in humans, dogs, and mice, $(18,19)$ in vitro metabolic studies using liver microsomes should evaluate both CYPs and UGT, suggesting that in vitro metabolic studies using hepatocytes may be a more convenient approach. However, as UGT contributes less to CAR elimination than to CYP in rats, $(19,20)$ the characteristic feature of CAR metabolism in rats have advantage in comparison of utility of liver microsomes and hepatocytes with plasma for evaluation of CAR elimination. It remains unclear whether liver microsomes and hepatocytes would be useful for the prediction of $C L_{\mathrm{h}, \text { in vivo }}$ of CAR. Thus, we compared the predicted $C L_{\mathrm{h}}$ of CAR using liver microsomes and hepatocytes from SD and CYP2Ddeficient DA rats as a model for human CYP2D6 PM. We also determined whether in vitro studies using liver microsomes and hepatocytes were useful for the prediction of stereospecific $C L_{\mathrm{h}}$.

\section{METHODS}

\section{Materials}

$R$-(+)- and $S$-(-)-CAR were obtained from Toronto Research Chemicals (Toronto, Canada). Propranolol hydrochloride, used as an internal standard for highperformance liquid chromatography (HPLC) analysis, and Hanks' Balanced Salt Solution were obtained from Sigma Aldrich (St. Louis, MO, USA). NADP $^{+}$, glucose-6-phosphate (G6P), and glucose-6phosphate dehydrogenase (G6PDH) were purchased from Oriental Yeast (Tokyo, Japan). All other chemicals and solvents were of HPLC grade or the highest purity available.

\section{Animal studies}

Male SD rats, 8 weeks of age and weighing between 250 and $290 \mathrm{~g}$, and male DA rats, 8 weeks of age and weighing between 140 and $180 \mathrm{~g}$, were obtained from Kiwa Laboratory Animals Co., Ltd. (Wakayama, Japan) and Shimizu Laboratory Supplies Co., Ltd. (Kyoto, Japan), respectively. Animals were housed in a humidity-controlled room at $24 \pm 2^{\circ} \mathrm{C}$ and had free access to water and a standard diet (MF, Oriental Yeast Co., Ltd., Tokyo). Animals were fasted overnight prior to experiments. CAR was dissolved in saline and administered as a single intravenous bolus at a dose of $4 \mathrm{mg} / \mathrm{kg}$. Blood samples $(250 \mu \mathrm{l}$ for each sample) were collected predose and at 5, 15,30,60, and 90 min after the dose via the jugular vein using syringes, placed in heparinized tubes, and immediately centrifuged at $6700 \mathrm{~g}$ for $10 \mathrm{~min}$ to obtain plasma. Animal experiments were conducted according to the Committee for the Care and Use of Laboratory Animals of the Faculty of Pharmacy of Kindai University (Osaka, Japan).

\section{Preparation of rat liver microsomes and hepatocytes}

Liver microsomes from SD and DA rats were prepared according to previously described methods.(21) Protein concentrations were determined using a BCA protein assay kit (Pierce Biotechnology, Inc., Rockford, IL, USA).

Hepatocytes were isolated from SD and DA rats by in situ perfusion of the liver with collagenase as reported by Seglen(22) and suspended in Hanks' Balanced Salt Solution.(21) Preparations with greater than $75 \%$ cell viability as determined by Trypan blue dye exclusion were used.

\section{Incubation of CAR enantiomers with rat liver microsomes and hepatocytes}

Incubation of rat liver microsomes or rat primary hepatocytes with $R$ - and $S$-CAR was conducted under the following conditions. The incubation mixture (total volume, $1 \mathrm{ml}$ ) contained $1 \mathrm{mg} / \mathrm{ml}$ of rat liver microsomes or $1 \times 10^{6}$ cells $/ \mathrm{ml}$ of hepatocytes in suspension, $100 \mathrm{mM}$ potassium phosphate buffer ( $\mathrm{pH} 7.4)$, an NADPH-generating system (1 mM NADP ${ }^{+}, 10 \mathrm{mM}$ glucose-6-phosphate, $1 \mathrm{IU} / \mathrm{ml}$ glucose-6-phosphate dehydrogenase, and 10 $\mathrm{mM}$ magnesium chloride), and $0.05-10 \mu \mathrm{M} R$ - or $S$ CAR. The reaction was initiated by adding $R$ - or $S$ CAR solution following a 5-min pre-incubation at $37^{\circ} \mathrm{C}$. At each time point, an aliquot of the reaction mixture $(100 \mu \mathrm{l})$ in rat liver microsomes or rat primary hepatocytes was placed in a centrifuge tube and the reaction was stopped by addition of $1 \mathrm{M}$ $\mathrm{NaOH}(100 \mu \mathrm{l})$. Seven milliliters of diethyl ether and $20 \mu \mathrm{l}$ of $2.5 \mu \mathrm{M}$ propranolol hydrochloride as an internal standard $(20 \mu \mathrm{l})$ were added and the mixture was shaken for $10 \mathrm{~min}$. After centrifugation at 1200 $\times g$ for $10 \mathrm{~min}$, the organic phase was removed and evaporated by vacuum evaporation, and the residue 
was dissolved immediately in $100 \mu$ l of methanol for HPLC analysis.

Determination of plasma protein and microsomal binding and blood to plasma concentration ratio Unbound fraction of plasma binding $\left(f_{\text {upplasma }}\right)$ and microsomal binding $\left(f_{u, m i c r o s o m e s}\right)$ were determined by equilibrium dialysis as reported previously.(21) Plasma or an in vitro incubation mixture containing rat liver microsomes and CAR $(10 \mu \mathrm{g} / \mathrm{ml}$ for plasma and $10 \mu \mathrm{M}$ for in vitro incubation mixture) without an NADPH-generating system were added to one chamber, and dialysis was performed at $37^{\circ} \mathrm{C}$ for $8 \mathrm{~h}$ using Spectra/Por MWCO 12-14,000 (Spectrum Japan, Ritto, Japan). In preliminary experiments, we confirmed that equilibrium was reached within this incubation period.

Whole blood to plasma concentration ratio $\left(R_{\mathrm{b}}\right)$ was determined by comparing the CAR concentration in plasma obtained from blood samples after a single oral dose of CAR $(20 \mathrm{mg} / \mathrm{kg})$.

\section{HPLC assay of CAR}

The concentrations of $R$ - and $S$-CAR were measured by HPLC (Liquid Chromatograph model LC-10AD; Shimadzu, Kyoto, Japan) with fluorescence detection (RF-10A fluorescence detector) according to the method reported by Rathod et al.(23) with some modifications.(24) For samples obtained from in vivo experiments, chromatographic separation was achieved using a Chiralcel OD-R column (4.6 × $250 \mathrm{~mm}, 10 \mu \mathrm{m}$, Daicel Corporation, Osaka, Japan) maintained at $40^{\circ} \mathrm{C}$. The mobile phase consisted of $50 \mathrm{mM}$ sodium acetate buffer ( $\mathrm{pH} 6.0$ ):acetonitrile $(25: 75, \mathrm{v}: \mathrm{v})$, and the flow rate was maintained at 1.0 $\mathrm{ml} / \mathrm{min}$. The excitation and emission wavelengths were set at $285 \mathrm{~nm}$ and $355 \mathrm{~nm}$, respectively. Standard curves for $R$ - and $S$-CAR were linear over the concentration range of $0.01-10 \mu \mathrm{M}$.

For samples obtained from in vitro experiments, chromatographic separation was achieved using an Inertsil ODS-3 column $(4.6 \times 100 \mathrm{~mm}, 4 \mu \mathrm{m}$, GL Sciences Inc., Tokyo, Japan) maintained at $40^{\circ} \mathrm{C}$. The mobile phase consisted of $100 \mathrm{mM}$ potassium phosphate buffer $(\mathrm{pH} 6.8)$ :methanol $(40: 60, \mathrm{v} / \mathrm{v})$, and the flow rate was maintained at $1.0 \mathrm{ml} / \mathrm{min}$. The excitation and emission wavelengths were set at 280 $\mathrm{nm}$ and $350 \mathrm{~nm}$, respectively. Standard curves for $R$ and $S$-CAR were linear over the concentration range of $0.01-10 \mu \mathrm{M}$.

\section{Data Analysis}

In vivo pharmacokinetic parameters were calculated by non-compartmental analysis using WinNonlin (Pharsight Co., Cary, NC, USA). Elimination halflife $\left(t_{1 / 2}\right)$ was calculated using $\ln 2 / k_{\mathrm{e}}$, where $k_{\mathrm{e}}$ is the elimination rate constant estimated by least-squares regression analysis of the plasma concentration-time curve. Area under the CAR concentration-time curve from time 0 to infinity $\left(A U C_{0-\infty}\right)$ was calculated by the trapezoidal rule using the determined values, which were extrapolated to infinity by dividing the last measured plasma concentration $\left(\mathrm{C}_{\text {last }}\right)$ by $k_{\mathrm{e}}$. Total clearance $\left(C L_{\mathrm{tot}}\right)$ was estimated by $\operatorname{dose} / A U C_{0-\infty}$. Volume of distribution at steady state $\left(V d_{\mathrm{ss}}\right)$ was calculated by multiplying the mean residence time (MRT), which was estimated by dividing the area under the first moment curve $\left(A U M C_{0-\infty}\right)$ by $A U C_{0-\infty}$, by $C L_{\text {tot. }}$.

Enzyme kinetic parameters were estimated using the substrate depletion assay in rat microsomes and hepatocytes as previously described.(11,21) Briefly, the time course of the percentage of remaining CAR was fitted to the first elimination function to estimate the initial substrate depletion rate constant $\left(K_{\text {dep }}\right)$. The first elimination function was determined by fitting the values at initial concentrations to the equation (1) using a non-linear least-squares regression analysis using WinNonlin:

$$
K_{d e p}=K_{d e p[S]=0} \cdot\left(1-\frac{s}{S+K_{m}}\right)
$$

$\mathrm{S}$ and $K_{\mathrm{dep}[\mathrm{S}]=0}$ are the substrate concentration and the theoretical maximum elimination rate constant at an infinitesimally low substrate concentration $\left(C L_{\text {int }}\right)$, respectively. The intrinsic clearance at an infinitesimally low substrate concentration $\left(C L_{\text {int }}\right)$ was calculated by dividing $K_{\mathrm{dep}[\mathrm{S}]=0}$ by the concentration of microsomal protein and hepatocytes. $C L_{\text {int }}$ values for liver microsomes and hepatocytes were expressed per kilogram of body weight by taking the weight of microsomes per gram of liver (45 mg protein/g liver) and the number of hepatocytes per gram of liver $\left(126 \times 10^{6}\right.$ cells $/ g$ liver), respectively, and liver weight per kilogram of body weight ( $40.4 \mathrm{~g}$ liver $/ \mathrm{kg}$ weight) to predict in vivo hepatic clearance $\left(C L_{\mathrm{h}}\right) \cdot(25,26) \quad C L_{\mathrm{h}}$ was estimated from $C L_{\text {int }}$ in liver microsomes based on the well-stirred model(27) as follows: 


$$
C L_{h}=\frac{Q_{h} \cdot \frac{f_{b}}{f_{u, i n c}} \cdot C L_{i n t}}{Q_{h}+\frac{f_{b}}{f_{u, i n c}} \cdot C L_{\text {int }}}
$$

$C L_{\mathrm{h}}$ was estimated from $C L_{\text {int }}$ in hepatocytes with (3) or without (4) plasma as follows:

$$
\begin{gathered}
C L_{h}=\frac{Q_{h} \cdot C L_{i n t}}{Q_{h}+C L_{i n t}} \\
C L_{h}=\frac{Q_{h} \cdot f_{b} \cdot C L_{i n t}}{Q_{h}+f_{b} \cdot C L_{i n t}}
\end{gathered}
$$

$Q_{\mathrm{h}}, f_{\mathrm{u}, \text { inc }}$, and $f_{\mathrm{b}}$ are hepatic blood flow, unbound fraction in the incubation mixture, and unbound fraction in the blood calculated by $f_{\text {uplasma }} / R_{\mathrm{b}}$, respectively.

The difference in the mean between the groups was analyzed statistically using Student's $t$-test.

\section{RESULTS}

\section{Pharmacokinetics of CAR after intravenous dosing}

Plasma concentrations of $R$ - and $S$-CAR after intravenous injection to SD and DA rats are shown in Figure 1, and the pharmacokinetic parameters are summarized in Table 1. Plasma concentrations of both CAR enantiomers were higher in DA rats than in SD rats. $R$-CAR concentrations were higher than $S$-CAR concentrations in both strains. $V d_{\text {ss }}$ and $C L_{\text {tot }}$ of $S$-CAR were approximately two times greater than those of $R$-CAR in both strains.

Enzymatic kinetics of CAR in rat liver microsomes and hepatocytes

Depletion profiles of $R$ - and $S$-CAR in liver microsomes of SD and DA rats are shown in Figure 2. The relationship between initial substrate concentration and $K_{\text {dep }}$ is shown in Figure 5a. Estimated kinetic parameters are summarized in Table 2. Although the calculated $C L_{\text {int }}$ for $S$-CAR was slightly higher than that for $R$-CAR in both strains, $C L_{\text {int }}$ of both enantiomers in DA rats was $42-$ $43 \%$ of that in SD rats.

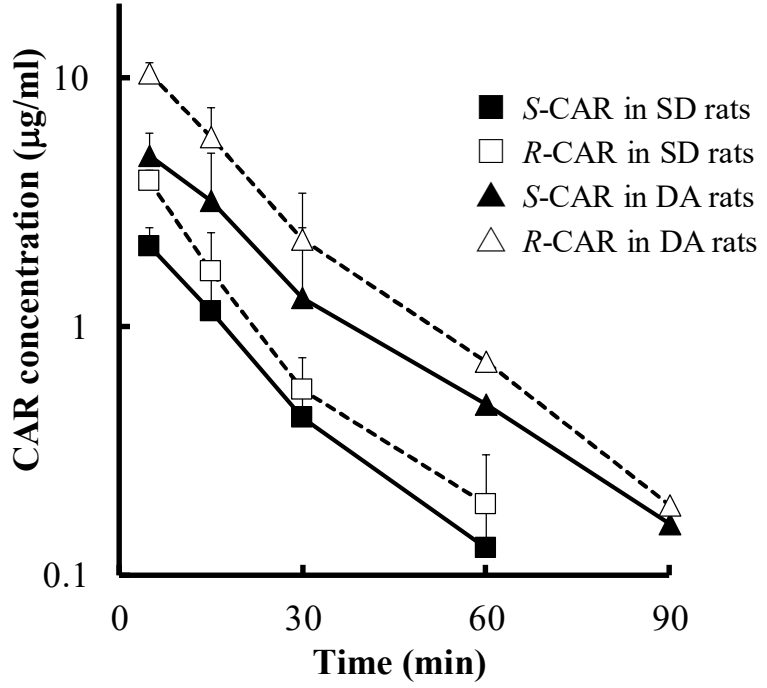

Figure 1. Plasma concentration versus time profiles of $R$ - and $S$-CAR $(4 \mathrm{mg} / \mathrm{kg})$ after intravenous injection to SD and DA rats. Data are expressed as the mean \pm S.D. $(n=3-4)$.

Depletion profiles of CAR enantiomers in hepatocytes of SD and DA rats and the relationship between initial substrate concentration and $K_{\text {dep }}$ are shown in Figures 3 and 5b, respectively, with kinetic parameters summarized in Table 3. $K_{\text {dep }}$ values obtained using hepatocytes from SD rats were strongly concentration-dependent, as shown using liver microsomes. However, $K_{\text {dep }}$ values obtained from DA rat hepatocytes, especially for $R$-CAR, were only slightly concentration-dependent. No marked differences were observed between the calculated $C L_{\text {int }}$ for $S$-CAR and $R$-CAR in SD rats. However, $C L_{\text {int }}$ for $S$-CAR in DA rats was 2.5 times higher than that for $R$-CAR. Thus, $C L_{\text {int }}$ of $R$ - and $S$ CAR in DA rats was $9.3 \%$ and $20 \%$, respectively, of $C L_{\text {int }}$ in $\mathrm{SD}$ rats.

Rat plasma was added to hepatocytes to investigate the effects of plasma protein binding. Depletion profiles of $R$ - and $S$-CAR in SD and DA rat hepatocytes in the presence of rat plasma, and the relationship between initial substrate concentration and $K_{\text {dep }}$ are shown in Figures 4 and 5c, respectively. Kinetic parameters are summarized in Table 4. $C L_{\text {int }}$ values in hepatocytes in the presence of plasma were $3-6 \%$ of those in the absence of plasma (Table 4 ) for both enantiomers and both strains, suggesting that plasma protein binding of CAR markedly decreased the free concentration of CAR. 
a)

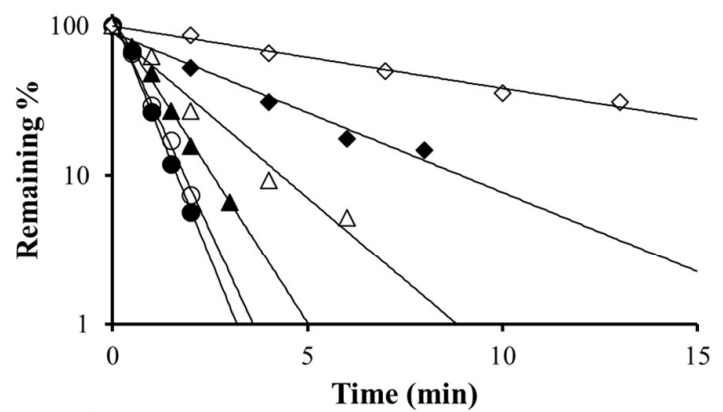

c)

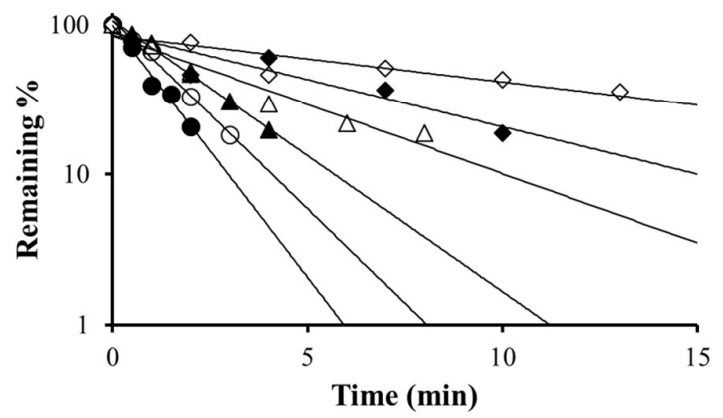

b)

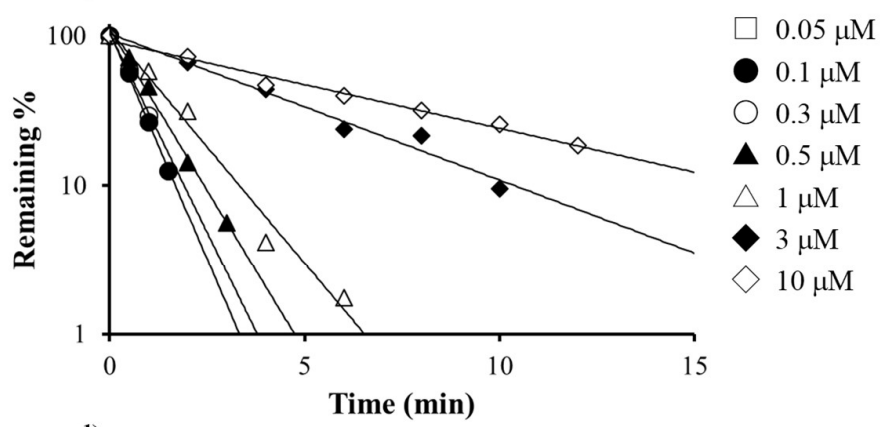

d)

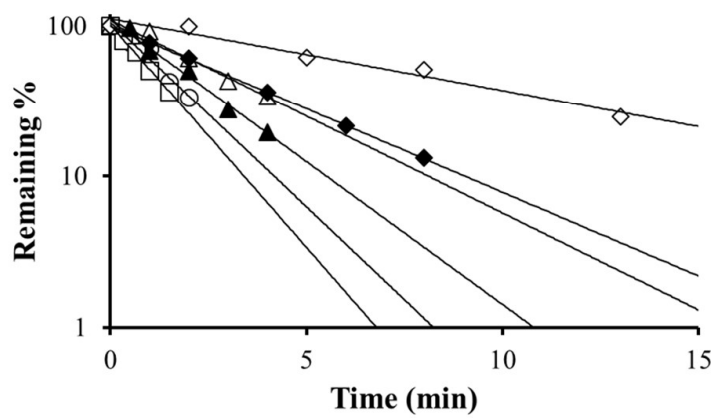

Figure 2. Depletion profiles of $\boldsymbol{R}$ - and $\boldsymbol{S}$-CAR in liver microsomes of SD and DA rats. a) $S$-CAR in SD rats, b) $R$ CAR in SD rats, c) $S$-CAR in DA rats, d) $R$-CAR in DA rats. The solid lines indicate fitted curves based on first-order elimination. Liver microsomes in each group were prepared from two rats. Data are expressed as the mean of duplicate values.

a)

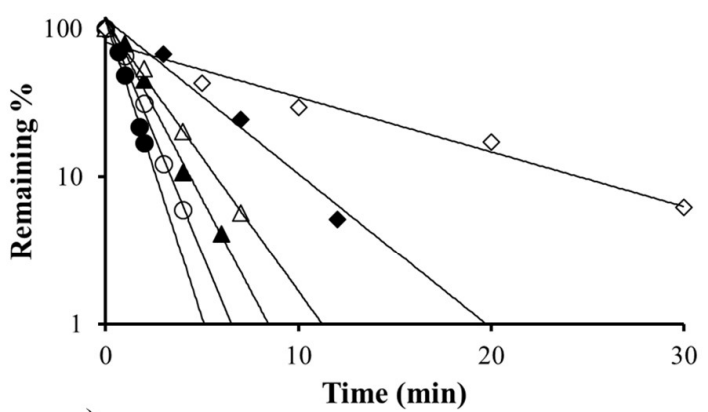

c)

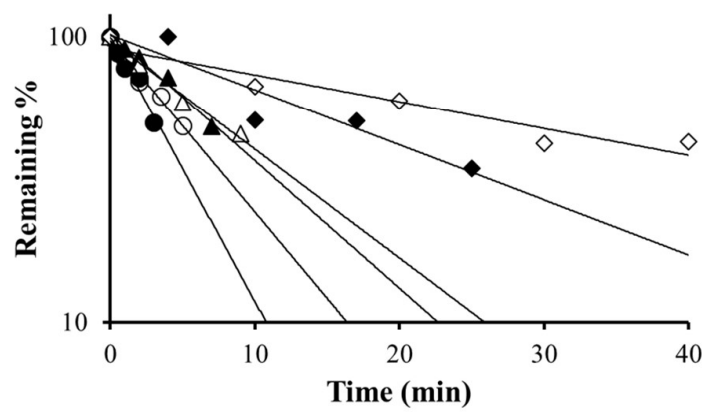

b)

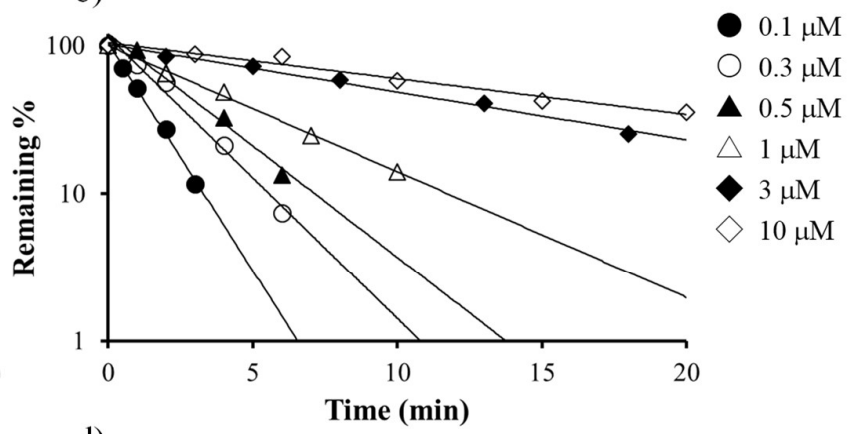

d)

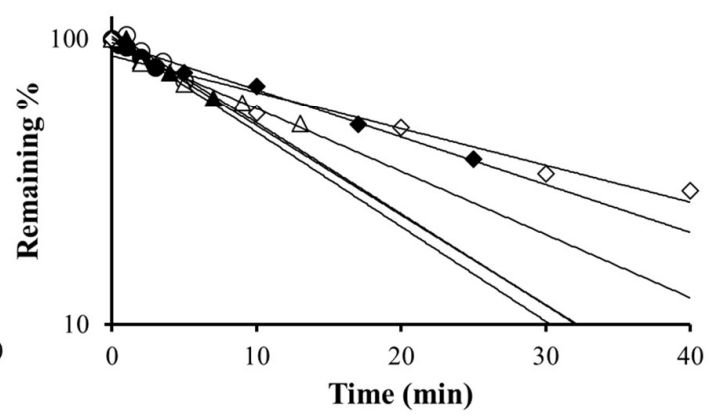

Figure 3. Depletion profiles of $\boldsymbol{R}$ - and $S$-CAR in hepatocytes in the absence of plasma of SD and DA rats. a) $S$-CAR in SD rats, b) $R$-CAR in SD rats, c) $S$-CAR in DA rats, d) $R$-CAR in DA rats. Solid lines indicate fitted curves based on first-order elimination. Hepatocytes in each group were prepared from two rats. Data are expressed as the mean of duplicate values. 

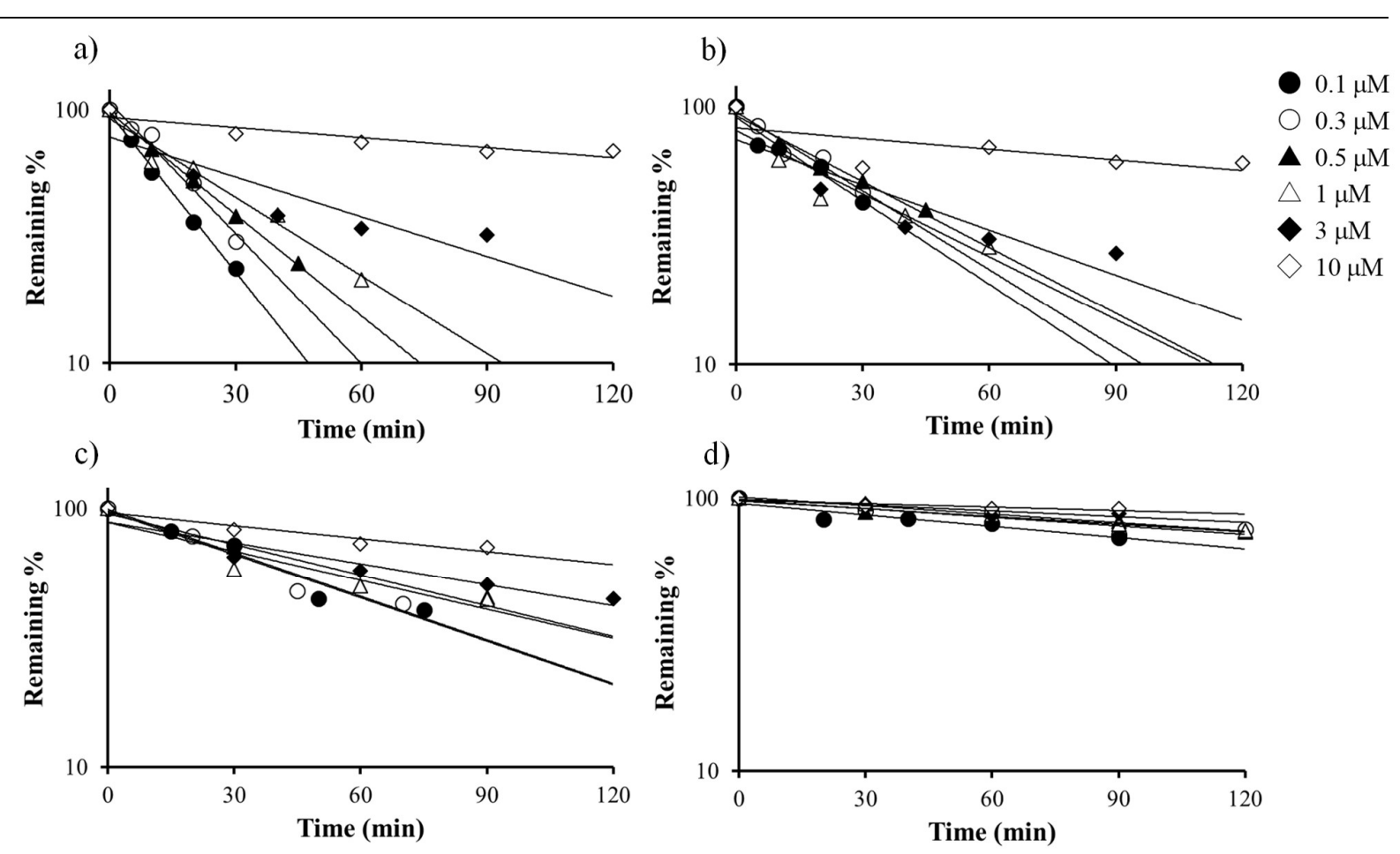

Figure 4. Depletion profiles of $\boldsymbol{R}$ - and $S$-CAR in hepatocytes in the presence of plasma of SD and DA rats. a) $S$ CAR in SD rats, b) $R$-CAR in SD rats, c) $S$-CAR in DA rats, d) $R$-CAR in DA rats. Solid lines indicate fitted curves based on the first-order elimination. Hepatocytes in each group were prepared from two rats. Data are expressed as the mean of duplicate values.
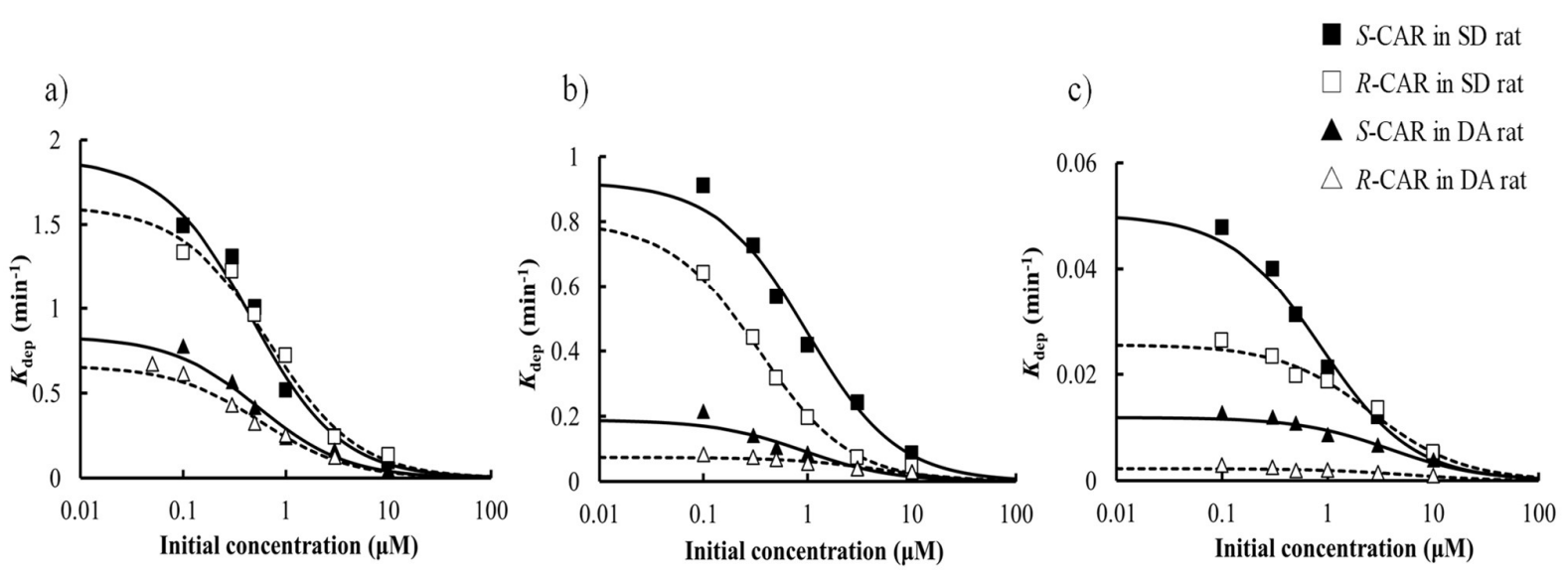

Figure 5. Relationships between initial substrate concentration and depletion rate constant of $\boldsymbol{R}$ - and $\boldsymbol{S}$-CAR in liver microsomes and hepatocytes of SD and DA rats. a) Liver microsomes. b) Hepatocytes in the absence of plasma. c) Hepatocytes in the presence of plasma. $\mathbf{m}$-CAR in SD rats, $\square: R$-CAR in SD rats, $\boldsymbol{\Delta}: S$-CAR in DA rats, $\triangle: R$-CAR in DA rats. Liver microsomes and hepatocytes in each group were prepared from two rats. Data are expressed as the mean of duplicate values. Solid and dashed lines represent the estimated curves as described in Materials and Methods.

Plasma protein and microsomal binding, and blood to plasma concentration ratio

The $f_{\mathrm{u}}$ values of $R$ - and $S$-CAR were $2.4-4.5 \%$ in both strains (Table 5). These values were comparable with the decrease in $C L_{\text {int }}$ values in hepatocytes in the presence of plasma (3-6\%, Table 4). The $f_{\mathrm{u}}$ value of $S$-CAR was 1.3 times greater than that of $R$-CAR in both strains. The $f_{\mathrm{u}}$ values in DA rats were 1.5 times greater than those in SD rats for both enantiomers. There were no differences in $R_{\mathrm{b}}$ 
and $f_{\mathrm{u}}$ in incubation mixtures containing rat liver microsomes between SD rats and DA rats.

\section{Estimation of hepatic clearance}

$C L_{\mathrm{h}}$ values were calculated from estimated $C L_{\text {int }}$ in rat liver microsomes and hepatocytes, as shown in Tables $2-4$ by using the well-stirred model. $C L_{\mathrm{h}}$ values estimated from experiments using rat liver microsomes were similar for both enantiomers and between $\mathrm{SD}$ and DA rats. Thus, $C L_{\mathrm{h}}$ calculated from in vitro experiments with liver microsomes from DA rats was different from estimates obtained using in vivo experiments (Table 1). In contrast, $C L_{\mathrm{h}}$ values predicted from in vitro experiments with DA rat hepatocytes were almost identical to those observed in vivo and were lower than $C L_{\mathrm{h}}$ values in $\mathrm{SD}$ rats, reflecting polymorphic pharmacokinetics in vivo. The relationship between predicted $C L_{\mathrm{h}}$ values from in vitro hepatocyte experiments with plasma correlated well with observed $C L_{\text {tot }}$ in vivo, which is expected to be very similar to $C L_{\mathrm{h}}\left(\mathrm{R}^{2}=0.7734\right)$ (Figure 6). The predicted $C L_{\mathrm{h}}$ values were within the range of unity $\pm 33 \%$.

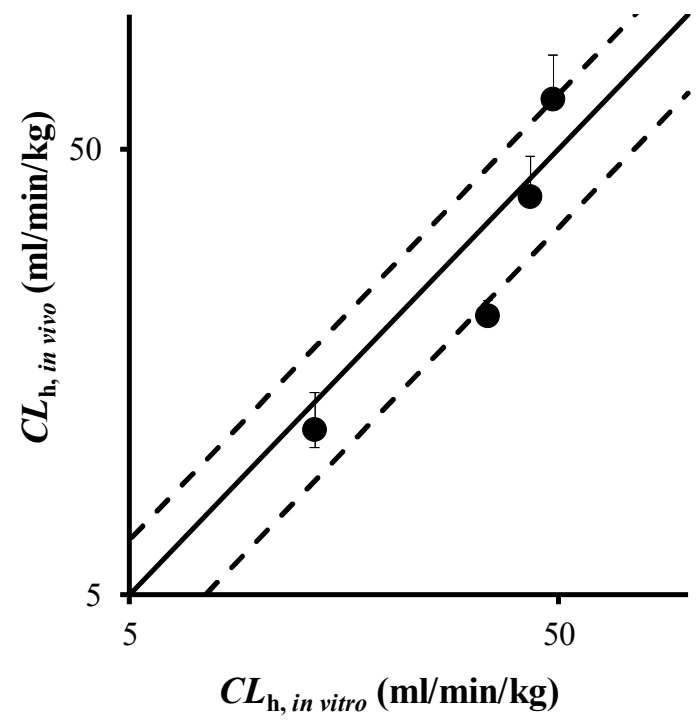

Fig. 6. Correlation between $C L_{\mathrm{h}}$ estimated from in vitro hepatocytes with plasma and those from the in vivo study. The vertical line shows $C L_{\mathrm{h} \text {,in vivo, }}$ which is expected to be very similar $C L_{\text {tot }}$ in Table 1 and the horizontal line shows $C L_{\mathrm{h}, \text { in }}$ vitro of hepatocytes with plasma in Table 5. The solid and dashed lines represent unity and unity $\pm 33 \%$, respectively.

\section{DISCUSSION}

In our previous study, the quantitative contribution of different CYP isoforms to enantioselective CAR metabolism was evaluated using a substrate depletion assay in humans. We demonstrated that CYP2D6 showed the highest affinity for $R$-CAR metabolism, followed by CYP3A4, CYP1A2, and CYP2C9. In contrast, metabolism of $S$-CAR is mainly mediated by CYP1 A2, followed by CYP2D6 and CYP3A4.(24) In rats, $R$-CAR was metabolized primarily by CYP2D2, followed by CYP3A2 and CYP3A1, whereas CYP2D1/2 and CYP3A1/2 catalyzed $S$-CAR metabolism.(12) Thus, the CYP2D subfamily is the main mediator of enzymatic $R$-CAR metabolism in both rats and humans. In contrast, $\mathrm{S}$ CAR is metabolized by CYP2D $1 / 2$ and CYP $3 A 1 / 2$ in rats and CYP1A2 in humans.

Plasma concentrations of $R$ - and $S$-CAR after intravenous injection of CYP2D-deficient DA rats were higher than those in SD rats, and the concentrations of $R$-CAR were higher than $S$-CAR in both strains. $V d_{\mathrm{ss}}$ and $C L_{\text {tot }}$ were greater for $S$-CAR than for $R$-CAR. These results were similar to previous reports of human pharmacokinetic characteristics of CAR enantiomers, $(2,3)$ confirming the possibility that DA and SD rats are suitable PM and EM animal models with respect to CYP2D6 activity. $(13,14)$

Although liver microsomes are commonly used for evaluation of CYP isoform(s) and for prediction of in vivo pharmacokinetics,(8-11) reports have shown that in vivo pharmacokinetic profiles of propranolol, but not metoprolol, in female DA rats were different from the profiles predicted in vitro using liver microsomes.(11) Previous studies in our laboratories have shown that rat hepatocytes are accurate predictors of in vivo pharmacokinetic profiles. $(16,17)$ Therefore, in the present study, prediction of $C L_{\mathrm{h}}$ of CAR, which is eliminated via plasma protein binding-sensitive stereoselective metabolism by the CYP2D subfamily, using liver microsomes and hepatocytes from SD and DA rats was evaluated to investigate which model is most useful for development of new candidate drugs. When predicted from SD and DA rat depletion profiles of $R$ - and $S$-CAR in rat liver microsomes, the calculated $C L_{\text {int }}$ for $S$-CAR was slightly higher than that for $R$-CAR in both strains. However, $C L_{\text {int }}$ in DA rats was $42-43 \%$ of $C L_{\text {int }}$ in $\mathrm{SD}$ rats for both enantiomers. In contrast, estimation of $C L_{\text {int }}$ using $\mathrm{SD}$ and DA rat hepatocytes showed no difference between $S$-CAR and $R$-CAR in SD rats. However, $C L_{\text {int }}$ for $S$-CAR in DA rats was 2.5 times higher than that for $R$-CAR. In addition, $C L_{\text {int }}$ of $R$ - and $S$-CAR in DA rats was estimated at $9.3 \%$ and $20 \%$ of $R$-CAR and $S$-CAR in SD rats, respectively. Furthermore, plasma protein binding affected uptake from blood to hepatic cells. The $f_{\mathrm{u}}$ values were comparable to the decrease in $C L_{\text {int }}$ values observed in hepatocytes in the presence of plasma. The $f_{\mathrm{u}}$ value of $S$-CAR was higher than that of $R$-CAR in both strains, and the 
values in DA rats were higher than those in SD rats for both enantiomers. Thus, the predicted $C L_{\mathrm{h}}$ values calculated from $C L_{\text {int }}$ in rat liver microsomes were similar both among enantiomers and between SD and DA rats. As such, $C L_{\mathrm{h}}$ calculated in vitro using liver microsomes from DA rats was different from in vivo results. In contrast, predicted $C L_{\mathrm{h}}$ values calculated from $C L_{\text {int }}$ in DA rat hepatocytes were similar to those observed in vivo and were lower than $C L_{\text {int }}$ values in SD rats, reflecting polymorphic pharmacokinetics in vivo. In addition, predicted $C L$ int values from in vitro hepatocyte studies correlated with the observed $C L_{\text {tot }}$ in vivo, which is expected to be very similar to $C L_{\mathrm{h}}$.

CAR is metabolized by UGT, including UGT2B7, UGT2B4, and UGT1A1, following hydroxylation in humans. $(28,29)$ Furthermore, CAR is metabolized by UGT in addition to CYPs in humans, dogs, and mice, but not rats. $(18,19)$ Although the mechanisms for stereoselective CAR metabolism in hepatocytes but not liver microsomes are unknown, possible explanations are as follows: (1) hydroxylated CAR metabolite(s), which inhibit CYP activities, might be eliminated via glucuronidation in rat hepatocytes, but not liver microsomes, and/or (2) CAR might be metabolized by UGT in rats as reported by Ishida et al. (30) that the glucuronidation of $R$ - and $S$-CAR was observed in rat liver microsomes. There are other possibilities that the differences of the accessibility of CAR to metabolic enzymes or CAR uptake between liver microsomes and hepatocytes could affect the CAR metabolism. Further detailed studies evaluating metabolism in rat hepatocytes are necessary. The relatively high $C L_{\text {int }}$ of CAR could contribute to a good relationship between predicted $C L_{\mathrm{h}}$ values from in vitro $C L_{\text {int }}$ from hepatocytes. A different result in the drug with low hepatic extraction ratio may be obtained, although the in vivo $C L_{\text {int }}$ of a low hepatic extraction ratio such as warfarin could be predicted from in vitro $C L_{\text {int }}$ from hepatocytes (31). It would be useful to further investigate whether the drugs with low hepatic extraction ratio as well as CAR show good relationships between predicted $C L_{\mathrm{h}}$ values from in vitro hepatocyte experiments with plasma and those with observed $C L_{\text {tot }}$ in vivo. In addition, in vitro metabolic studies using hepatocytes are expected to be more convenient than using liver microsomes depending on the species differences of metabolism. In vitro metabolic studies using human hepatocytes would be of particular interest and value.

In conclusion, substrate depletion assay using hepatocytes rather than liver microsomes was useful for the prediction of $C L_{\mathrm{h}, \text { in vivo }}$ of $\mathrm{CAR}$, leading the possibility that this approach could be apply the prediction of $C L_{\mathrm{h}, \text { in }}$ vitro of the other drugs metabolized by CYPs and phase II metabolic enzymes.

\section{CONFLICT OF INTEREST}

The authors declare no conflict of interest.

\section{ACKNOWLEDGMENTS}

This work was supported by the "Antiaging" Project for Private Universities, with a matching fund subsidy from the Japanese Ministry of Education, Culture, Sports, Science and Technology (MEXT). This research was also supported in part by the MEXT-Supported Program for the Strategic Research Foundation at Private Universities, 20142018 (S1411037).

\section{REFERENCES}

1. Fisker FY, Grimm D, Wehland M. Third-Generation Beta-Adrenoceptor Antagonists in the Treatment of Hypertension and Heart Failure. Basic Clin Pharmacol Toxicol [Internet]. 2015 Jul;117(1):5-14. Available from: http://doi.wiley.com/10.1111/bcpt.12396

2. Neugebauer G, Akpan W, von Möllendorff E, Neubert P, Reiff K. Pharmacokinetics and disposition of carvedilol in humans. J Cardiovasc Pharmacol [Internet]. 1987;10 Suppl 11:S85-8. Available from: http://www.ncbi.nlm.nih.gov/pubmed/2454375

3. Neugebauer G, Akpan W, Kaufmann B, Reiff K. Stereoselective disposition of carvedilol in man after intravenous and oral administration of the racemic compound. Eur J Clin Pharmacol [Internet]. 1990;38 Suppl 2:S108-11. Available from: http://www.ncbi.nlm.nih.gov/pubmed/1974498

4. Zhou HH, Wood AJ. Stereoselective disposition of carvedilol is determined by CYP2D6. Clin Pharmacol Ther [Internet]. 1995 May;57(5):518-24. Available from: http://doi.wiley.com/10.1016/00099236(95)90036-5

5. Stoschitzky K, Koshucharova G, Lercher P, Maier R, Sakotnik A, Klein W, et al. Stereoselective effects of (R)- and (S)-carvedilol in humans. Chirality [Internet]. 2001 Jul;13(7):342-6. Available from: http://doi.wiley.com/10.1002/chir.1042

6. Bartsch W, Sponer G, Strein K, Müller-Beckmann B, Kling L, Böhm E, et al. Pharmacological characteristics of the stereoisomers of carvedilol. Eur J Clin Pharmacol [Internet]. 1990;38 Suppl 2:S104-7. Available

from: http://www.ncbi.nlm.nih.gov/pubmed/1974497

7. Oldham HG, Clarke SE. In vitro identification of the human cytochrome P450 enzymes involved in the metabolism of R(+)- and S(-)-carvedilol. Drug Metab Dispos [Internet]. 1997 Aug;25(8):970-7. Available from:

http://www.ncbi.nlm.nih.gov/pubmed/9280405

8. Suzuki A, Iida I, Tanaka F, Akimoto M, Fukushima $\mathrm{K}$, Tani M, et al. Identification of human cytochrome 
$\mathrm{P}-450$ isoforms involved in metabolism of $\mathrm{R}(+)$ - and S(-)-gallopamil: utility of in vitro disappearance rate. Drug Metab Dispos [Internet]. 1999 Nov;27(11):1254-9. Available from: http://www.ncbi.nlm.nih.gov/pubmed/10534309

9. Obach RS, Reed-Hagen AE. Measurement of Michaelis constants for cytochrome P450-mediated biotransformation reactions using a substrate depletion approach. Drug Metab Dispos [Internet]. 2002 Jul;30(7):831-7. Available from: http://www.ncbi.nlm.nih.gov/pubmed/12065442

10. Niwa T, Shiraga T, Ishii I, Kagayama A, Takagi A. Contribution of human hepatic cytochrome p450 isoforms to the metabolism of psychotropic drugs. Biol Pharm Bull [Internet]. 2005 Sep;28(9):1711-6. Available http://www.ncbi.nlm.nih.gov/pubmed/16141545

11. Komura H, Kawase A, Iwaki M. Application of substrate depletion assay for early prediction of nonlinear pharmacokinetics in drug discovery: assessment of nonlinearity of metoprolol, timolol, and propranolol. J Pharm Sci [Internet]. 2005 Dec;94(12):2656-66. Available from: http://linkinghub.elsevier.com/retrieve/pii/S0022354 916319098

12. Iwaki M, Niwa T, Nakamura Y, Kawase A, Komura H. Relative contribution of rat CYP isoforms responsible for stereoselective metabolism of carvedilol. J Toxicol Sci [Internet]. 2018;43(1):5963. Available from: https://www.jstage.jst.go.jp/article/jts/43/1/43_59/_a rticle

13. Saito K, Sakai N, Kim H-S, Ishizuka M, Kazusaka A, Fujita S. Strain differences in diazepam metabolism at its three metabolic sites in sprague-dawley, brown norway, dark agouti, and wistar strain rats. Drug Metab Dispos [Internet]. 2004 Sep;32(9):959-65. Available

from: http://www.ncbi.nlm.nih.gov/pubmed/15319337

14. Kawase A, Fujii A, Negoro M, Akai R, Ishikubo M, Komura H, et al. Differences in cytochrome P450 and nuclear receptor mRNA levels in liver and small intestines between SD and DA rats. Drug Metab Pharmacokinet [Internet]. 2008;23(3):196-206. Available http://www.ncbi.nlm.nih.gov/pubmed/18574324

15. Komura $H$, Iwaki $M$. NONLINEAR PHARMACOKINETICS OF PROPAFENONE IN RATS AND HUMANS: APPLICATION OF A SUBSTRATE DEPLETION ASSAY USING HEPATOCYTES FOR ASSESSMENT OF NONLINEARITY. Drug Metab Dispos [Internet]. 2005 Mar 2;33(6):726-32. Available from: http://www.ncbi.nlm.nih.gov/pubmed/15743979

16. Komura $\mathrm{H}$, Iwaki $\mathrm{M}$. Pharmacokinetics and metabolism of metoprolol and propranolol in the female DA and female Wistar rat: the female DA rat is not always an animal model for poor metabolizers of CYP2D6. J Pharm Sci [Internet]. 2005 Feb;94(2):397-408. Available from: http://linkinghub.elsevier.com/retrieve/pii/S0022354 916316914

17. Komura H, Iwaki M. Usefulness of hepatocytes for evaluating the genetic polymorphism of CYP2D6 substrates. Xenobiotica [Internet]. 2005 Jun 22;35(6):575-87. Available from: http://www.tandfonline.com/doi/full/10.1080/00498 250500202056

18. Neugebauer G, Neubert P. Metabolism of carvedilol in man. Eur $\mathrm{J}$ Drug Metab Pharmacokinet [Internet].;16(4):257-60. Available from: http://www.ncbi.nlm.nih.gov/pubmed/1823868

19. Schaefer WH, Politowski J, Hwang B, Dixon F, Goalwin A, Gutzait L, et al. Metabolism of carvedilol in dogs, rats, and mice. Drug Metab Dispos [Internet]. 1998 Oct;26(10):958-69. Available from: http://www.ncbi.nlm.nih.gov/pubmed/9763400

20. Fujimaki M, Shintani S, Hakusui H. Stereoselective metabolism of carvedilol in the rat. Use of enantiomerically radiolabeled pseudoracemates. Drug Metab Dispos [Internet].;19(4):749-53. Available http://www.ncbi.nlm.nih.gov/pubmed/1680650

21. Uno S, Fujii A, Komura H, Kawase A, Iwaki M. Prediction of metabolic clearance of diclofenac in adjuvant-induced arthritis rats using a substrate depletion assay. Xenobiotica [Internet]. 2008 May 22;38(5):482-95. Available from: http://www.tandfonline.com/doi/full/10.1080/00498 250801935982

22. Seglen PO. Preparation of isolated rat liver cells. Methods Cell Biol [Internet]. 1976;13:29-83. Available from: http://www.ncbi.nlm.nih.gov/pubmed/177845

23. Rathod R, Prasad LPC, Rani S, Nivsarkar M, Padh H. Estimation of carvedilol in human plasma by using HPLC-fluorescence detector and its application to pharmacokinetic study. J Chromatogr B Analyt Technol Biomed Life Sci [Internet]. 2007 Oct 1;857(2):219-23. Available from: http://linkinghub.elsevier.com/retrieve/pii/S1570023 207005120

24. Iwaki M, Niwa T, Bandoh S, Itoh M, Hirose $H$, Kawase A, et al. Application of substrate depletion assay to evaluation of CYP isoforms responsible for stereoselective metabolism of carvedilol. Drug Metab Pharmacokinet [Internet]. 2016 Dec;31(6):425-32. Available from: https://linkinghub.elsevier.com/retrieve/pii/S134743 6716300702

25. Boxenbaum $H$. Interspecies variation in liver weight, hepatic blood flow, and antipyrine intrinsic clearance: extrapolation of data to benzodiazepines and phenytoin. J Pharmacokinet Biopharm [Internet]. 1980 Apr;8(2):165-76. Available from: http://www.ncbi.nlm.nih.gov/pubmed/6107379

26. Iwatsubo T, Hirota N, Ooie T, Suzuki H, Sugiyama Y. Prediction of in vivo drug disposition from in vitro data based on physiological pharmacokinetics. Biopharm Drug Dispos [Internet]. 1996 May;17(4):273-310. Available from: http://doi.wiley.com/10.1002/\%28SICI\%291099081X\%28199605\%2917\%3A4\%3C273\%3A\%3AAI D-BDD961\%3E3.0.CO\%3B2-R

27. Pang KS, Rowland M. Hepatic clearance of drugs. I. Theoretical considerations of a \&quot;well- 
stirred\&quot; model and a \&quot;parallel tube\&quot; model. Influence of hepatic blood flow, plasma and blood cell binding, and the hepatocellular enzymatic activity on hepatic drug clearance. J Pharmacokinet Biopharm [Internet]. 1977 Dec;5(6):625-53. Available http://www.ncbi.nlm.nih.gov/pubmed/599411

28. Ohno A, Saito Y, Hanioka N, Jinno H, Saeki M, Ando $\mathrm{M}$, et al. Involvement of human hepatic UGT1A1, UGT2B4, and UGT2B7 in the glucuronidation of carvedilol. Drug Metab Dispos [Internet]. 2004 Feb 1;32(2):235-9. Available from: http://dmd.aspetjournals.org/cgi/doi/10.1124/dmd.3 2.2.235

29. Takekuma Y, Takenaka T, Yamazaki K, Ueno K, Sugawara M. Stereoselective metabolism of racemic carvedilol by UGT1A1 and UGT2B7, and effects of mutation of these enzymes on glucuronidation activity. Biol Pharm Bull [Internet]. 2007 Nov;30(11):2146-53. Available from: http://www.ncbi.nlm.nih.gov/pubmed/17978490
30. Ishida K, Taira S, Morishita H, Kayano Y, Taguchi M, Hashimoto Y. Stereoselective oxidation and glucuronidation of carvedilol in human liver and intestinal microsomes. Biol Pharm Bull [Internet]. 2008 Jun;31(6):1297-300. Available from: http://www.ncbi.nlm.nih.gov/pubmed/18520073

31. Griffin SJ, Houston JB. Prediction of in vitro intrinsic clearance from hepatocytes: comparison of suspensions and monolayer cultures. Drug Metab Dispos [Internet]. 2005 Jan;33(1):115-20. Available from:

http://www.ncbi.nlm.nih.gov/pubmed/15608354

32. Kumar S, Samuel K, Subramanian R, Braun MP, Stearns RA, Chiu S-HL, et al. Extrapolation of diclofenac clearance from in vitro microsomal metabolism data: role of acyl glucuronidation and sequential oxidative metabolism of the acyl glucuronide. J Pharmacol Exp Ther [Internet]. 2002 Dec 1;303(3):969-78. Available from: http://jpet.aspetjournals.org/cgi/doi/10.1124/jpet.102 .038992 
J Pharm Pharm Sci (www.cspsCanada.org) 22, 72 - 84, 2019

\section{TABLES}

Table 1. Kinetic parameters of $R$ - and $S$-CAR after intravenous injection to SD and DA rats.

\begin{tabular}{|c|c|c|c|c|}
\hline \multirow{2}{*}{ Parameters } & \multicolumn{2}{|c|}{ SD } & \multicolumn{2}{|c|}{ DA } \\
\hline & $R$ & $S$ & $R$ & $S$ \\
\hline$C_{0}(\mu \mathrm{g} / \mathrm{ml})$ & $5.5 \pm 0.9$ & $2.9 \pm 0.5$ & $14.8 \pm 1.1$ & $6.7 \pm 0.4$ \\
\hline$A U C_{0-\infty}(\mu \mathrm{g} \min / \mathrm{ml})$ & $80.7 \pm 19.5$ & $51.6 \pm 11.1^{*}$ & $264 \pm 58$ & $142 \pm 11^{*}$ \\
\hline$t_{1 / 2}(\min )$ & $13.8 \pm 3.4$ & $14.9 \pm 3.0$ & $15.9 \pm 2.6$ & $17.4 \pm 2.4$ \\
\hline$V d_{\mathrm{ss}}(1 / \mathrm{kg})$ & $0.39 \pm 0.09$ & $0.70 \pm 0.08^{* *}$ & $0.14 \pm 0.02$ & $0.30 \pm 0.01^{* *}$ \\
\hline$k_{\mathrm{e}}\left(\min ^{-1}\right)$ & $0.05 \pm 0.01$ & $0.05 \pm 0.01$ & $0.04 \pm 0.01$ & $0.04 \pm 0.01$ \\
\hline$C L_{\text {tot }}(\mathrm{ml} / \mathrm{min} / \mathrm{kg})$ & $25.9 \pm 6.0$ & $40.4 \pm 10.5$ & $7.8 \pm 1.7$ & $14.1 \pm 1.1^{* *}$ \\
\hline$C L_{\text {tot }}(\mathrm{ml} / \mathrm{min} / \mathrm{kg})^{\text {a) }}$ & $39.0 \pm 9.0$ & $64.5 \pm 16.7$ & $11.7 \pm 2.5$ & $21.1 \pm 1.7^{* *}$ \\
\hline
\end{tabular}

Each value represents the means \pm S.D. $(n=3-4)$.

Significant different $(* ; p<0.05$ and $* * ; p<0.01)$ from corresponding $R$-CAR.

a) Blood total clearance of $R$ - and $S$-CAR.

Table 2. Kinetic parameters of $R$ - and $S$-CAR metabolism in liver microsomes of SD and DA rats.

\begin{tabular}{|c|c|c|c|c|}
\hline \multirow{2}{*}{ Parameters } & \multicolumn{2}{|c|}{ SD } & \multicolumn{2}{|c|}{ DA } \\
\hline & $R$ & $S$ & $R$ & $S$ \\
\hline$K_{\mathrm{dep}[\mathrm{s}]=0}\left(\min ^{-1}\right)$ & $1.61 \pm 0.17$ & $1.89 \pm 0.20$ & $0.66 \pm 0.05$ & $0.84 \pm 0.11$ \\
\hline$K_{\mathrm{m}}(\mu \mathrm{M})$ & $0.68 \pm 0.12$ & $0.46 \pm 0.07$ & $0.58 \pm 0.09$ & $0.54 \pm 0.12$ \\
\hline$C L_{\text {int }}(\mu \mathrm{l} / \mathrm{min} / \mathrm{mg}$ protein $)$ & $1612 \pm 165$ & $1891 \pm 196$ & $663 \pm 52$ & $836 \pm 113$ \\
\hline$C L_{\text {int }}(\mathrm{ml} / \mathrm{min} / \mathrm{kg})$ & $2930 \pm 300$ & $3438 \pm 356$ & $1206 \pm 94$ & $1519 \pm 206$ \\
\hline
\end{tabular}

The data represent the estimate \pm standard error obtained from MULTI program. 
Table 3. Kinetic parameters of $R$-and $S$-CAR metabolism in hepatocytes in the absence or presence of plasma of SD and DA rats.

\begin{tabular}{|c|c|c|c|c|c|c|c|c|}
\hline \multirow{3}{*}{ Parameters } & \multicolumn{4}{|c|}{ SD } & \multicolumn{4}{|c|}{ DA } \\
\hline & \multicolumn{2}{|c|}{$R$} & \multicolumn{2}{|c|}{$S$} & \multicolumn{2}{|c|}{$R$} & \multicolumn{2}{|c|}{$S$} \\
\hline & -plasma & + plasma & -plasma & + plasma & -plasma & + plasma & -plasma & + plasma \\
\hline$K_{\mathrm{dep}[\mathrm{s}]=0}\left(\min ^{-1}\right)$ & $0.80 \pm 0.14$ & $0.026 \pm 0.002$ & $0.92 \pm 0.05$ & $0.050 \pm 0.005$ & $0.075 \pm 0.005$ & $0.0024 \pm 0.0002$ & $0.19 \pm 0.02$ & $0.012 \pm 0.0008$ \\
\hline$K_{\mathrm{m}}(\mu \mathrm{M})$ & $0.34 \pm 0.09$ & $2.62 \pm 0.35$ & $0.97 \pm 0.10$ & $0.87 \pm 0.17$ & $4.95 \pm 1.18$ & $5.96 \pm 1.73$ & $0.92 \pm 0.20$ & $3.89 \pm 0.78$ \\
\hline$C L_{\text {int }}\left(\mu \mathrm{l} / \mathrm{min} / 10^{6}\right.$ cells $)$ & $801 \pm 140$ & $25.6 \pm 1.4$ & $923 \pm 52$ & $50.3 \pm 5.4$ & $75.4 \pm 5.5$ & $2.4 \pm 0.2$ & $189 \pm 22$ & $11.9 \pm 0.8$ \\
\hline$C L_{\text {int }}(\mathrm{ml} / \mathrm{min} / \mathrm{kg})$ & $4047 \pm 706$ & $129 \pm 7$ & $4661 \pm 263$ & $254 \pm 27$ & $380 \pm 28$ & $11.9 \pm 1.0$ & $956 \pm 109$ & $60.3 \pm 4.2$ \\
\hline
\end{tabular}

The data represent the estimate \pm standard error obtained from MULTI program.

Table 4. Physiological parameters of $R$ - and $S$-CAR in SD and DA rats.

\begin{tabular}{lccccc}
\hline \multirow{2}{*}{ Parameters } & \multicolumn{2}{c}{ SD } & & \multicolumn{2}{c}{$\mathrm{DA}$} \\
\cline { 2 - 3 } \cline { 5 - 6 } & $0.66 \pm 0.04$ & $0.63 \pm 0.01$ & & $0.67 \pm 0.04$ & $0.67 \pm 0.08$ \\
\hline$R_{\mathrm{b}}$ & $0.024 \pm 0.003$ & $0.030 \pm 0.003^{*}$ & & $0.035 \pm 0.004$ & $0.045 \pm 0.005^{*}$ \\
$f_{\text {u,plasma }}$ & $0.27 \pm 0.03$ & $0.28 \pm 0.03$ & & $0.23 \pm 0.04$ & $0.23 \pm 0.04$ \\
$f_{\mathrm{u}, \text { inc,microsome }}$ & & & & & 55 \\
$Q_{\mathrm{h}}(\mathrm{ml} / \mathrm{min} / \mathrm{kg})^{\text {a) }}$ & 55 & & & & \\
\hline
\end{tabular}

Each value represents the means \pm S.D. $(n=3-4)$.

Significant different $(* ; p<0.05)$ from corresponding $R$-CAR.

a) Data are from Kumar et al. (32). 
Table 5. In vitro $C L_{\mathrm{h}}$ of $R$ - and $S$-CAR predicted in liver microsomes and hepatocytes of SD and DA rats.

\begin{tabular}{lccccc}
\hline & \multicolumn{4}{c}{ Predicted $C L_{\mathrm{h}}$ from in vitro data $(\mathrm{ml} / \mathrm{min} / \mathrm{kg})$} \\
\cline { 2 - 4 } \multicolumn{1}{c}{ Tissue } & $R$ & & & \multicolumn{3}{c}{$\mathrm{DA}$} \\
\cline { 2 - 3 } \cline { 5 - 6 } & 48.4 & 50.3 & & 45.9 & 48.8 \\
Liver microsomes & 40.2 & 44.1 & & 14.6 & 29.5 \\
Hepatocytes (-plasma) & 42.9 & 48.4 & & 13.5 & 34.1 \\
Hepatocytes (+plasma) & & & & & \\
\hline
\end{tabular}

